entorno como mejor manera de relativizar la singularidad del curso de los acontecimientos en el caso español. Las referencias escogidas para el contraste son la I República portuguesa, surgida en 1910, y la III República francesa de los años 1930. Esta tercera apoyatura de su análisis es, a nuestro juicio, la más endeble de las tres. Las referencias a dichas experiencias republicanas dan la sensación de ser pinceladas esporádicas que no consiguen del todo servir de referencias comparativas. Además, y sin cuestionar la oportunidad ni validez en la selección de esos casos, no hubiese estado de más volver la mirada a los casos italiano y alemán, donde en esos años fascismo, nacionalsocialismo, socialismo y comunismo intentaban hacer valer en la calle su definición de la nación, de su nación, en un clima de polarización ideológica extrema y de crisis de la democracia.

Jesús CASQUETE

\section{Cómo mejorar la preparación de los encuestadores}

Normalmente suelo comenzar las recensiones con una breve explicación sobre los motivos de tal elección. Cuando era estudiante de Sociología, allá por la década de los ochenta, trabajé como encuestador en Bilbao. Eran años de gran convulsión política, y de muchas encuestas que pretendían medir tal convulsión, y muy pronto pude apreciar los problemas que aparecían cuando se realizaban «determinadas preguntas», las reticencias que mostraban los entrevistados cuando eran preguntados por determinados aspectos políticos.

Ante esta situación, y con el fin de conocer con precisión las funciones de este trabajo, traté de documentarme con libros que explicaran la labor del encuestador. Me quedé sorprendido de los escasos materiales publicados sobre el tema, y pensé que el problema estaba en que yo no había sabido buscarlos. La práctica, me dijeron entonces, era la que hacía «buenos encuestadores», era la que diferenciaba el buen del mal encuestador. Con todo, quedé sorprendido que en ningún lugar aparecieran recogidas las experiencias de los buenos encuestadores. Con el paso del tiempo me he dado cuenta que apenas existen materiales sobre esta temática ${ }^{1}$, y por este motivo me ha parecido conveniente realizar una recensión sobre un «manual audiovisual sobre encuestadores» titulado Cómo mejorar las tasas de respuesta. Una guía para los entrevistadores en el acceso al entrevistado (How to Improve Survey Response Rates. A guide for Interviewers on the Doorstep).

Bajo este título, la editorial Sage comercializa un documento audiovisual (un vídeo y una guía

\footnotetext{
1 A principios del año 2005 únicamente existen en nuestro idioma tres publicaciones monográficas sobre el tema (Manzano et al., 1996; Díaz de Rada, 2001a y 2005).
} 
sobre cómo utilizarlo) elaborado por el National Centre for Social Research (1999a y 1999b) para la formación de sus encuestadores. El vídeo se centra específicamente en cómo el encuestador puede evitar la no respuesta total en las encuestas, un tema de máxima actualidad en el ámbito de la investigación social, como han puesto de manifiesto -entre otros-Bradburn (1992), Smith (1995: 157), Goves y Couper (1998), Groves et al. (2002) y Stoop (2005).

Un indicador de la importancia de esta temática es la afirmación realizada en la sesión de clausura de la reunión internacional de la Asociación Americana para la Investigación de la Opinión Pública (AAPOR), donde se señaló que el mayor problema que presentará la investigación mediante encuesta en el siglo XXI será el incremento de la no respuesta (Bradburn, 1992: 391397). La importancia de este hecho queda también patente al revisar un estudio realizado a empresas de investigación de mercados de los Estados Unidos, donde se señala que los dos mayores problemas que tendrá el sector en los próximos diez años serán el incremento en la tasa de rechazo y la presencia de contestadores automáticos para filtrar Ilamadas telefónicas (Smith, 1995: 157). La presencia de este problema en las últimas publicaciones de la colección
Survey Mehodology Section, de la editorial Wiley (Groves y Couper, 1998; Groves et al., 2002; Biemer y Lyberg, 2003), una editorial de referencia en el ámbito de la investigación social; unido a los dos números monográficos sobre no respuesta de la revista Journal of Official Statistics (1999 y 2001), sin duda una de las más prestigiosas en el ámbito de la investigación mediante encuestas, son otros indicadores de la importancia - y la gravedad — de este problema.

De las diferentes formas de mitigar la influencia de la no respuesta total, el vídeo del National Centre se centra en la más efectiva, evitar la no respuesta aumentando la formación de los encuestadores. Es la más efectiva en la medida que un encuestador bien preparado puede hacer que prácticamente desaparezca la no respuesta; pero también la más difícil por la necesidad de motivar y preparar a un equipo humano que, en muchas ocasiones, considera el trabajo de encuestador como una ocupación temporal hasta que se encuentra un trabajo mejor².

Los expertos en la materia señalan que a nivel general existen dos comportamientos cuando se establece el contacto con una persona con el fin de entrevistarla ${ }^{3}$ : el primero tiene como objetivo primordial maximizar el número de

2 Este aspecto ha sido analizado en detalle por Wert (1996: 44) en sus referencias a la necesaria profesionalización del encuestador y, más recientemente, por Fernández Esquinas (2003: 47-77).

3 Buscando un término que defina con precisión a la persona que es requerida para contestar a una encuesta, hemos rechazado algunos de los nombres más utilizados en la actualidad puesto que únicamente recogen a las personas que colaboran con la investigación (encuestados, entrevistados, etc.), obviando así a las personas que no colaboran. Este motivo nos lleva a buscar un término que defina la capacidad o la opción de responder, razón por la cual utilizaremos la palabra «respondiente» para referirnos tanto a las personas que son entrevistadas como a aquellas que por diversos motivos no lo son. 
aceptaciones por unidad de tiempo, mientras que el segundo tiene como fin maximizar la probabilidad de participación de cada respondiente. El primer comportamiento genera unas elevadas tasas de no respuesta, puesto que se fundamenta en realizar el mayor número de entrevistas en el mínimo espacio de tiempo. Esto implica que el tiempo empleado en cada entrevista debe ser el mínimo posible, provocando, en ocasiones, rápidas explicaciones sobre los objetivos de la investigación, el proceso de selección, etc., que producen un gran número de recelos de cooperar y rechazos de cooperación. El segundo comportamiento trata de aumentar la probabilidad de respuesta de la persona que ha respondido a la llamada del encuestador. La cantidad de tiempo empleado para conseguir cada entrevista es algo que no se tiene en cuenta, puesto que se persigue minimizar el ratio viviendas contactadas/viviendas que cooperan. La ausencia de preocupación por el tiempo permite realizar una mejor explicación de los objetivos de la investigación, aclarando las dudas suscitadas por el respondiente, al tiempo que posibilita la utilización de las estrategias de persuasión expuestas en párrafos anteriores. Más que preocuparse por incrementar la probabilidad del «sí», está interesada en evitar el «no deseo cooperar». Este comportamiento se fundamenta en la idea que a medida que la interacción aumenta es más difícil para un interlocutor «despedir» al otro interlocutor (Groves y Couper, 1998: 38-39).

El vídeo del National Centre for Social Research es el resultado de varias investigaciones (Campanelli, 1997, y Morton-Williams, 1991) realizadas por el Centro y que llegan a la con- clusión que los entrevistadores con más experiencia están mejor preparados, presentan menores tasas de rechazo y son más conscientes que los respondientes pueden encontrarse ligeramente ansiosos ante la visita de alguien que inesperadamente solicita su cooperación para «responder a unas preguntas». Los entrevistadores experimentados se adaptan mejor a las características de los posibles entrevistados, abandonando rápidamente el protocolo de presentación establecido al reemplazarlo por mensajes (verbales y no verbales) más apropiados y sutiles que reducen el rechazo (National Centre for Social Research, 1999: 3-4).

Los autores del vídeo insisten en que estas habilidades —adquiridas con la experiencia práctica en el trabajo de campo- pueden enseñarse, si bien es difícil de hacerlo vía métodos convencionales; y por este motivo proponen la necesidad de hacerlo mediante un documento audiovisual que muestre cómo deben comportarse los entrevistadores a la hora de contactar con una vivienda. El vídeo -a juicio del National Centre for Social Research- es probablemente el mejor medio para mostrar las estrategias y protocolos de acceso a la vivienda, en la medida que se muestran situaciones reales sobre cómo deben proceder los entrevistadores.

Llama la atención, a este respecto, la escasa atención que ha prestado la literatura especializada a la recogida de información. Este olvido podría justificarse porque la entrevista «cuantitativa» no precisa gran pericia en el desarrollo de la misma, al ser un protocolo estandarizado, pero ¿qué ocurre con el acceso a la vi- 
vienda? ${ }^{4}$. Resulta sorprendente que los libros de metodología apenas hayan dedicado espacio a este aspecto. Algunos olvidan totalmente el trabajo de campo, que es realmente el «responsable» de la información recogida (la materia prima de la investigación), mientras que otros reducen la información sobre el trabajo de campo a la selección del número de entrevistadores y en cómo llevar a cabo una correcta formación. La práctica totalidad de los textos se olvidan de las estrategias a emplear a la hora de acceder a la vivienda, como demuestran Hox y DeLeeuw (2002: 103) tras analizar la formación que reciben los encuestadores de 32 países: únicamente los encuestadores suecos reciben entrenamiento sobre la no respuesta, y este entrenamiento ocupa - tan sólo-el 35\% de su formación.

En definitiva, el vídeo resuelve un problema fundamental (y escasamente investigado) de la investigación social actual, tratando de mitigar la influencia de la no respuesta por la eliminación de ésta. Una de las ventajas de esta forma de proceder es que puede utilizarse conjuntamente con otras estrategias como aumentar el número de contactos, utilización de gratificaciones, insistencia en el anonimato y confidencialidad de las respuestas, sustitución de los que no responden, volver a entrevistar a los que han rechazado responder, realizar un segundo muestreo entre los que no responden, ponderaciones de la muestra... y otros métodos que hemos presentado en otro trabajo (Díaz de Rada, 2000: 65-127).

En cuanto a la estructura del trabajo objeto de esta recensión, se trata de un vídeo de $30 \mathrm{mi}$ nutos de duración protagonizado por cuatro entrevistadores y varios actores que actúan como entrevistados. El vídeo está dividido en $15 \mathrm{sec}-$ ciones, cada una referida a las distintas situaciones que tienen lugar en el momento en el que el entrevistador accede a una determinada vivienda ${ }^{5}$ : 1) preparación;2) acceso a la vivienda; 3) comportamiento en la puerta de la vivienda (comportamiento general); 4) llamar en un mal momento; 5) comportamiento en la puerta de la vivienda, asegurarse que la dirección es correcta; 6) comportamiento en la puerta de la vivienda, explicación de la encuesta; 7) cómo tratar con «porteros automáticos»; 8) ¿por qué yo?, llama a mi vecino; 9) selección de la persona a entrevistar dentro de la vivienda; 10) no interesado; 11) comienzo de la entrevista; 12) obstáculos diversos; 13) cómo comportarse con la gente mayor; 14) buenos días y malos días en el trabajo diario del entrevistador; y 15) recomendaciones generales relativas a la importancia de comportarse ade-

\footnotetext{
4 No debemos olvidar el planteamiento fundamental de la encuesta muestral, que permite reflejar adecuadamente la realidad cuando es respondida por muestras probabilísticas, esto es, por muestras donde todo el mundo tiene la misma probabilidad de ser elegido. Dicho de otro modo, la capacidad de acceso y localización de toda la población es el fundamento metodológico esencial de la encuesta.

5 Los contenidos de cada una serán presentados en detalle más adelante.
} 
cuadamente. Dentro de cada sección pueden diferenciarse tres partes:

a) Cada sección comienza con una «situación real», esto es, una filmación de un entrevistador y un entrevistado en un momento del acceso a la vivienda (por ejemplo, llamando a la puerta, explicando el objetivo de la entrevista, etc.).

b) Posteriormente, los encuestadores comentan esta «situación real» (la situación filmada anteriormente) y otras situaciones similares.

c) Un texto con una serie de recomendaciones referidas a esta situación pone fin a cada sección.

Aunque ésta es la estructura básica de cada sección, en ocasiones sufre variaciones al desaparecer la primera parte, la «situación real». Esto sucede, por ejemplo, en las tres primeras secciones: en la primera, que lleva por título preparación, los entrevistadores comentan las estrategias que llevan a cabo cada vez que comienza un nuevo estudio, y la situación real es sustituida por imágenes donde los entrevistadores se preparan antes de llevar a cabo la primera entrevista ${ }^{6}$ : comprobando su aspecto en el espejo del coche, ordenando todos los materiales, etc.

En la segunda sección, titulada acceso a la vivienda, se muestran varias recomendaciones sobre la conveniencia de aparcar el coche lejos de la vivienda a entrevistar y la importancia de recorrer la zona andando para conocer el lugar e intuir el tipo de personas que viven en esa zona ${ }^{7}$. En el vídeo se insiste en que el tipo de edificaciones, las personas que van por la calle, etc., pueden proporcionar una importante información («proporcionan pistas») sobre si hay personas en la vivienda y en qué horarios suelen estar en casa. Como indicadores de esta situación pueden considerarse la existencia de varias botellas de leche en la puerta, buzón lleno de cartas, manchas de aceite en el lugar donde se aparca el coche, etc. En definitiva, se trata de «recoger pistas» para que cada llamada realizada sea lo más efectiva posible; es decir, que cada llamada logre una entrevista ${ }^{8}$.

El acceso a la vivienda continúa con el comportamiento en la puerta de la vivienda, cuyos contenidos se refieren a cómo colocarse en la entrada de la vivienda para que la presencia del entrevistador sea lo menos agresiva posi-

\footnotetext{
${ }^{6}$ Los entrevistadores solos, es decir, sin entrevistados.

7 It's funny how with some houses... you do the feeling of...

8 Conviene tener en cuenta que los entrevistadores del National Centre for Social Research realizan -al menos- cuatro visitas a una vivienda y, de éstas, al menos una se realiza por la noche o durante el fin de semana (National Centres for Social Research, 1999c: 19). Por este motivo, el «acceso a la vivienda» tiene como objetivo la búsqueda de «pistas» para reducir el número de visitas.
} 
ble al que abre la puerta ${ }^{9}$, así como la presentación a realizar cuando se encuentra frente al respondiente. Se señala con claridad que la mejor presentación es la más breve ${ }^{10}$; y esto se explica porque el respondiente puede estar mirando al entrevistador pero no escucharle: la situación real que reproduce el vídeo —en esta sección- es un entrevistado que está pensando «qué me querrá vender este pesado».

Estas tres primeras secciones se fundamentan en los comentarios de los entrevistadores, ilustradas con algunas imágenes en las que se reproducen las situaciones de las que ellos hablan, y es a partir de este momento cuando cada sección aparece dividida en las tres partes que apuntamos más atrás: situación real, comentarios y recomendaciones.

Llamar en un mal momento es el título de la cuarta sección, donde se muestra un entrevistador que - tras realizar la presentación al residente de una vivienda- oye a un niño llorar y procede -rápidamente- a decir al respondiente que es un mal momento para sus propósitos y que volverá en otro momento. En la discusión posterior los entrevistadores presentan diversos indicadores que muestran que es un mal momento para las personas seleccionadas (nerviosismo o agitación del que abre la puer- ta, olor a comida, reunión familiar, etc.), y comentan sobre la importancia que sea el propio entrevistador el que tome la iniciativa de posponer la entrevista ${ }^{11}$. La sección termina con un texto donde se presentan las siguientes recomendaciones:

«Hay personas que están muy ocupadas. Cuando se constate que se ha realizado una visita en un mal momento, es conveniente "retirarse voluntariamente" antes de que lo solicite el entrevistado:

- Muchos rechazos son circunstanciales.

- Para la mayor parte de las personas es más fácil decir "no" que "no ahora".

- Es importante estar atento al lenguaje del cuerpo y otras señales "no verbales".

- No presiones a cooperar. Retírate antes de que el entrevistado manifieste un rechazo.

Sé breve, no digas mucho del objetivo de la investigación. Siempre podrás hablar con el entrevistado después de la entrevista».

La quinta sección está referida al comportamiento en la puerta de la vivienda, centrado

9 Téngase en cuenta que no siempre el que abre la puerta será la persona a entrevistar.

10 ... the best introduction is the one which is a brief one...

11 La situación en nuestro país es la contraria, tal y como se desprende de las investigaciones realizadas por Camacho y otros (2000). 
específicamente en asegurarse que se trata de la dirección correcta. El hecho de preguntar a los residentes que confirmen si esta dirección es correcta busca implicar a los respondientes, enviándoles un mensaje sobre la importancia de entrevistar a las personas que ahí viven, la importancia de la selección de esta vivienda. Esta pregunta busca también «implicar» al respondiente, finalizando así el «monólogo» del entrevistador. En este momento el objetivo del entrevistador es que el entrevistado pase de una actitud pasiva a una actitud activa: observando el carné del entrevistador para que constate que éste es quien dice ser, respondiendo al entrevistador cuando le pregunta si la dirección es correcta, leyendo la carta de presentación del director de la investigación, etc.

A continuación el entrevistador debe proceder con la explicación de la encuesta; uno de los momentos de más tensión puesto que —con el fin de paliar la situación de sorpresa del respondiente- el entrevistador debe proporcionar una situación de tranquilidad, mostrando el carné de entrevistador y otros materiales que den «seriedad» a su visita. En este momento el vídeo muestra con nitidez una situación habitual para las personas que han trabajado como encuestadores: hay un momento de silencio después de la presentación del entrevistador, mo- mento en el que el entrevistador está esperando la respuesta del respondiente; aunque también pueden producirse dudas que se transforman en preguntas del tipo: «¿de qué trata el estudio?», «lo siento, no me interesa», «¿cuánto dura la entrevista?», etc. Sin embargo, la mayor parte de las veces el respondiente está observando al entrevistador «esperando» qué se demanda de él. Normalmente, los entrevistadores novatos comienzan a hablar en este momento, proporcionando demasiadas explicaciones y justificaciones de su comportamiento. En realidad, lo que debe hacerse es «dar una breve pista de lo próximo que va a suceder» (National Research for Social Research, 1999b: 19-20); por ejemplo, tomar la carpeta con la encuesta y decir «¿comenzamos?, ¿qué te parece si procedemos?».

Aunque en la sociedad inglesa no son muy frecuentes los porteros automáticos (la mayor parte de la población vive en viviendas unifamiliares), se agradece que el vídeo contemple también esta situación tan habitual en nuestro país ${ }^{12}$. Así, la sección 7 se dedica a cómo tratar con porteros automáticos, que son definidos como «la ruina de la vida del entrevistador» (p. 21), por la ausencia de comunicación no verbal que dificulta una correcta interacción ${ }^{13}$.

\footnotetext{
12 La primera oleada de la Encuesta Social Europea, por ejemplo, ha detectado que el 67,2\% de la población entrevistada vive en pisos y/o apartamentos. Los residentes en estas viviendas son, por otro lado, los que presentan la menor tasa de cooperación (Cuxart y Riba, 2005: 37).

13 Según los expertos en comunicación, un tercio de la información es transmitida por las palabras, otro 33\% con la entonación, y otro tercio con los gestos. Gestos que en esta situación están ausentes.
} 
En el vídeo se elige una situación en la que el portero automático presenta algunos problemas de funcionamiento (algo, por otra parte, no muy infrecuente), con mucho ruido en la calle y un respondiente que dice no oír nada. Otro aspecto relevante de esta situación es que el encuestador tiene la oportunidad de «colarse» en el edificio aprovechando que alguien sale y deja la puerta abierta. Esta situación, que sucede muy frecuentemente en la actividad diaria de cualquier encuestador ${ }^{14}$, es resuelta con gran «profesionalidad» por el entrevistador-actor, que, tras pensar «quizás debiera entrar, es una tentación», rechaza hacerlo ${ }^{15}$. Esta acción se justifica (en la p. 21 del libro-guía) por el fuerte impacto que puede tener la presencia de un entrevistador (al que no se le espera) en el rellano de la casa.

Tras superar la barrera del portero automático, el entrevistador llama a la puerta de la vivienda del respondiente y comienza explicando el motivo de su visita. Esta sección está dedicada a convencer a la persona para que acceda a ser entrevistada, y lleva por título ¿por qué yo?, llama a mi vecino. Aquí se apela a cómo proceder para que desaparezcan en el respondiente las reticencias a cooperar. La situación utilizada es un entrevistado que no tiene trabajo, que está cobrando el subsidio de desempleo, y teme que la entrevistadora sea alguien de la oficina del paro que le esté contro- lando. La entrevistadora explica el motivo de la visita, el respondiente sigue mostrando sus reticencias a cooperar, y sugiere al entrevistador visite a sus vecinos ya que ellos están trabajando. En esta situación el entrevistador señala que es necesario conseguir la colaboración de personas diferentes: de todas las edades, todas las opiniones, todas las formas de vida..., es decir, un adecuado «resumen» de toda la sociedad.

Tras acceder a la vivienda es necesario realizar una selección de la persona a entrevistar dentro de la vivienda, aspecto que centra el interés de la novena sección. Se utiliza un método de selección muy similar al propuesto por Kish en 1949, si bien presenta menos «intrusividad» que éste al preguntar por las iniciales de los nombres de los residentes en esa vivienda; en vez de por el sexo, edad y ocupación, como procede el método de Kish (Kish, 1965: 398). La mayor parte de las investigaciones realizan una selección dentro de la vivienda con el fin de eliminar el sesgo «de la persona que abre la puerta»; es decir, que no se seleccione siempre a los que abren (Díaz de Rada, 2001a: 130-134). Normalmente, esa situación es desconocida para la persona que reside en la vivienda visitada, que asume que cualquier miembro de la vivienda puede ser entrevistado, de modo que será necesario explicar por qué se procede de esta forma; y por

14 Como han demostrado, en nuestro país, Camacho y otros (2000).

15 Maybe I should just go in... it is so tempting... oh, better not. 
este motivo el vídeo recomienda realizar tal explicación a la persona que abre la puerta. Esta situación justifica por qué conviene no dar mucha información sobre el tema de la investigación al que abre la puerta, puesto que es posible que no sea la persona a entrevistar.

Tras seleccionar a la persona dentro de la vivienda se pregunta por ésta para proceder con la entrevista. La sección número 10 utiliza la misma situación mostrada en la sección anterior, con la diferencia que -en este caso- el entrevistador se enfrenta con que la persona seleccionada es diferente a la que abrió la puerta. El título de esta sección da cuenta de la situación a abordar: no interesado. En este caso la persona seleccionada no desea cooperar y vuelve a llamar al que abrió la puerta. Éste se justifica diciendo que a él normalmente le gustan «este tipo de cosas». El entrevistador debe emplear aquí sus recursos para explicarle por qué debe ser entrevistada la persona elegida; que una vez seleccionada no es posible cambiar. En este ejemplo, y ante las reticencias continuadas del respondiente, decide aplazar la entrevista concertando una cita para dentro de unos días.

En estas situaciones, el vídeo recomienda conversar con los respondientes para conocer las razones de su falta de interés con el fin de insistir en los motivos por los que esta encuesta puede ser interesante para ellos. En ocasiones, el entrevistador puede conocer algo sobre los residentes en la vivienda (presencia de niños, gente mayor, etc.) y mencionar partes del cuestionario que pueden ser de especial interés: por ejemplo, si hay preguntas sobre el colegio, cuidado de los niños, pensiones, etc.
El vídeo advierte también que el entrevistador debe recordar que los respondientes no saben las preguntas que deberán responder; y que en ocasiones dicen «no estar interesados» como una forma de protegerse de intrusos con preguntas embarazosas. «Nadie quiere parecer estúpido, y el hecho de tomar parte en una investigación social puede ser intimidatorio para algunos colectivos» (pp. 25-26 del libro-guía). Estos recelos no declarados deben ser eliminados por el entrevistador. La mejor forma para que la gente se sienta segura de su capacidad para participar es «romper el hielo» aludiendo a la sencillez de la tarea. En el vídeo, por ejemplo, una entrevistadora señala su estrategia: «... Siempre les digo que no voy a preguntar sobre aspectos de los que no conocen las respuestas... Se trata de sus opiniones... Podrías comenzar para que veas que es así... Si no te gusta lo dejamos...».

Con el paso del tiempo se van superando los obstáculos para acceder al respondiente, y la sección 11 ya transcurre dentro de la vivienda. Aquí se analiza cómo actuar inmediatamente antes de proceder con la entrevista: explicando el propósito del estudio, recordando la confidencialidad de las respuestas, y señalando que todas las respuestas serán analizadas y mostradas de forma agregada. En las recomendaciones sobre esta sección se señala que debe responderse con honestidad a las preguntas del entrevistado. No puedo dejar de preguntarme por la honestidad de las respuestas de estos encuestadores cuando los respondientes pregunten por la duración de la entrevista; si tenemos en cuenta que en el informe elaborado con los cuestionarios empleados en el vídeo se señala que el tiempo medio en res- 
ponder al cuestionario superó los 60 minutos (Park et al., 2001: 266).

Por otro lado, es muy sorprendente la situación mostrada en el vídeo, con un entrevistador dentro de la vivienda del respondiente (en el salón). Resulta asombroso cuando lo comparo con la situación de nuestro país. De acuerdo que varios manuales anglosajones recomiendan hacer la entrevista en el interior de la vivienda (entre otros, Institute for Social Research, 1976); ahora bien, mi experiencia como encuestador - contrastada con otros colegas - me lleva a afirmar que la mayor parte de las entrevistas en nuestro país se hacen en el umbral de la puerta; y son escasas las ocasiones en las que el entrevistado permite acceder a su vivienda.

Volviendo al vídeo, la sección 12 se dedica a obstáculos diversos, entre los que destacan los sentimientos de inferioridad de los estratos socialmente más bajos cuando un entrevistador llega con un ordenador, presenta un gran dominio del lenguaje, etc. A estas alturas tan sólo quedan tres secciones, dedicadas a cómo realizar entrevistas a personas de la tercera edad (sección 13); buenos días y malos días en la actividad diaria del entrevistador (sección 14), y varias recomendaciones generales sobre cómo comportarse.
Respecto a cómo comportarse con las personas pertenecientes a la tercera edad, se insiste en que el entrevistador debe tener sumo cuidado en el momento del acceso a la vivienda. Es necesario mostrar el carné de identificación del encuestador y explicar con suma claridad ${ }^{16}$ los motivos de la visita. Tras la entrevista conviene dejar alguna documentación (carta del instituto de investigación, tarjeta o alguna hoja con el anagrama de la organización, etc.) para parientes o amigos que deseen conocer algo más sobre el entrevistador y las razones de su visita.

Cuando estas personas vacilan o tienen dudas para tomar parte en la investigación conviene no presionar para conseguir la entrevista en ese momento «al precio que sea». Es mejor fijar una cita cuando esté con algún pariente, amigo o vecino que le tranquilice. Esta situación particular aparece reflejada en el vídeo. La respondiente señala que es visitada por su hija todos los fines de semana y, como el entrevistador aprecia que se muestra reticente a cooperar, decide aplazar la entrevista hasta el día en que venga su hija. Se señala también que cuando se vaya a visitar una vivienda de ancianos ${ }^{17}$ conviene presentarse al portero antes de proceder con la búsqueda de la persona seleccionada.

En la penúltima sección, dedicada a los buenos días y malos días en el trabajo diario del entre-

\footnotetext{
16 Se busca evitar dificultades auditivas. No debemos olvidar que algunos sentidos, como el oído, comienzan a resentirse a partir de una determinada edad.

17 Se refiere a pisos construidos específicamente para ancianos, no residencias.
} 
vistador, se alude a cómo reaccionar ante los malos días, días con muchos rechazos y en los que no se consigue ninguna entrevista. Cuando el entrevistador tiene un mal día (con muchos rechazos o ausencias), lo mejor es tomarse un descanso para hacer algo diferente y divertido (sentarse en el coche a escuchar música, leer un libro, tomar un café o acudir a un museo, dar un paseo, etc.). Seguir trabajando tras esta situación es contraproducente; puesto que el desánimo y la falta de confianza son percibidos por los respondientes, lo que reduce su posibilidad de participar, creando así un círculo vicioso.

Por último, el vídeo termina con una serie de recomendaciones generales relativas a la importancia de comportarse adecuadamente y nunca perder los buenos modales. El entrevistador nunca debe olvidarse de agradecer al entrevistado su atención y su tiempo, al tiempo que recomiendan dar una tarjeta de visita del instituto (National Centre for Social Research) para que la siguiente vez que sea visitado por un entrevistador conozca sus demandas y sepa el tipo de preguntas que deberá responder.

En la guía del vídeo se señala que es muy frecuente que los entrevistadores con poca experiencia se muestren nerviosos a la hora de preguntar a alguien si desea tomar parte en un sondeo. Uno de los entrevistadores señala que esta situación no es necesariamente una desventaja, en la medida que numerosos respon- dientes reaccionan - ante una persona nerviosa- con un deseo de ayudarle. Por otro lado, y basado en el hecho que numerosos rechazos son circunstanciales, este entrevistador recomienda volver a visitar a las personas que han rechazado responder. En muchas ocasiones, éstas acceden a ser entrevistadas puesto que el rechazo pudo estar originado porque tenían un mal día o porque el entrevistador llegó en mal momento.

De esta forma termina el vídeo, de treinta minutos de duración, que ha aportado mucho sobre el trabajo de campo al redactor de estas líneas, y eso que he tenido una dilatada experiencia como encuestador. Si a un encuestador le ha aportado mucho, ¿qué no puede aportar a los que se enfrentan a este trabajo por primera vez? Esto me lleva a recomendar utilizar este trabajo en sesiones de formación de entrevistadores. Acostumbrados a las explicaciones orales, o a la autoformación mediante la lectura de «manuales de instrucciones de campo», la utilización del vídeo resulta una innovación didáctica de gran interés. Resulta sorprendente que la difusión masiva de los reproductores de vídeo en nuestras sociedades ${ }^{18}$ no haya estado acompañada de un mayor aprovechamiento de este recurso para la enseñanza de métodos y técnicas de investigación.

Otras ventajas están relacionadas con el instrumento empleado, el vídeo, que permite un

\footnotetext{
18 Mucho más cuando consideramos la facilidad actual para elaborar estos documentos con la tecnología digital.
} 
mejor reflejo de las situaciones. Frente a la tradicional explicación de «situaciones diversas» en manuales de encuestadores, el vídeo añade mucha más realidad al proceso de formación del entrevistador, al tiempo que ayuda a prestar atención a pequeños -y numerosos- detalles fundamentales para conseguir una mayor cooperación. El vídeo, además, presenta una mayor facilidad para comprender cómo resolver las situaciones habituales con las que se encuentra un encuestador.

Otro aspecto tremendamente interesante de este documento es que muestra la gran profesionalización del encuestador en la sociedad inglesa: el hecho de emplear cartas de presentación de la investigación, una carta de la policía local justificando la presencia del entrevistador, etc., son algunos indicadores del cuidado que los investigadores del National Centre ponen en la realización de los trabajos de campo.

Por último, el hecho que se haya realizado en la sociedad inglesa, una sociedad socialmente «más fría» que la nuestra, ¿no puede garantizar un mayor éxito en la sociedad mediterránea?, mucho más dada a la «charla». Si este vídeo funciona en Inglaterra, ¿cómo no va a funcionar aquí?
Expuestas las virtudes, consideramos que este trabajo presenta también algunos problemas: en primer lugar, la barrera del idioma, que no se trata tanto de la comprensión del inglés, sino los registros idiomáticos particulares que utilizan esos encuestadores (inglés de bajo nivel cultural, inglés «hindú», etc.). Este hecho, señalado como inconveniente por una persona que no tiene el inglés como lengua de origen, puede ser visto también como una ventaja cuando se considera la interculturalidad de la sociedad inglesa. Ahora bien, esta interculturalidad presenta un enorme inconveniente cuando consideramos una de las recomendaciones más importantes sobre los rasgos de los entrevistadores. Nos referimos, concretamente, al consejo de Groves y Couper (1998: 35) —sin duda, dos de los mayores expertos mundiales en el tema- cuando recomiendan que "los entrevistadores deben ser lo más parecidos posible a la población que es encuestada»19. Este hecho, verdaderamente difícil de cumplir en una sociedad tan diversa como la inglesa, es reflejado en el vídeo al utilizar encuestadores con diferentes registros idiomáticos. Digamos, en este sentido, que el vídeo enfatiza el componente didáctico al mostrar la «peor situación posible»; esto es, tomar como protagonistas entrevistadores de diferentes razas y condiciones sociales ${ }^{20}$.

\footnotetext{
19 «Cuando los rasgos de los entrevistadores son similares a los de los respondientes la colaboración es mayor» (Groves y Couper, 1998: 35).

20 Como «peor situación posible» nos referimos a que hubiera sido mucho más sencillo presentar entrevistadores similares al «inglés medio», pero el vídeo ha buscado las situaciones más difíciles, colectivos «minoritarios» dentro de la sociedad inglesa.
} 
Un vídeo dirigido a los encuestadores ingleses debe recoger - lógicamente - la gran variedad cultural que existe en el país, y es muy interesante que sus protagonistas utilicen los diferentes registros idiomáticos presentes en esa sociedad; pero supone una gran dificultad para que investigadores de otros países puedan comprender el vídeo ${ }^{21}$.

Ahora bien, desde nuestro punto de vista, hubiera sido más didáctico si se hubieran utilizado un mayor número de «situaciones reales» y menos "charla entre entrevistadores", en la medida que las situaciones reales de la calle hacen más comprensibles las tareas que deben realizarse en el trabajo de campo. En la charla entre los entrevistadores se relatan situaciones tremendamente interesantes, aunque en algunas ocasiones son difíciles de percibir con nitidez por la propia situación en la que se produce esta charla: conversan demasiado rápido, en ocasiones hablan varias personas a la vez, etc. Consideramos que el vídeo hubiera ganado notablemente si estas situaciones hubieran sido presentadas mediante una «situación real».

Por último, una breve nota para hablar de los resultados de este trabajo, en el contexto para el que fue creado. Me consta que este vídeo ha sido utilizado para la formación de los encuestadores que participan en el British Social Atti- tudes, investigación que logró —en la edición del 2001- una tasa de respuesta del 62\% (Park y otros, 2001: 267). Lejos de lo que pudiera parecer, y como se ha demostrado en otros trabajos (Díaz de Rada, 2000, 2001b, 2004; Sánchez Carrión, 2000), esta tasa supera ampliamente la cooperación media lograda en las investigaciones que se realizan en nuestro país y en otros contextos similares (DeLeeuw, 1992; Hox y DeLeeuw, 2002: 111; Stoop, 2005: 23-32).

Como conclusión final, la publicación de este vídeo me parece un acierto y una suerte para todos los que estamos preocupados en la calidad de la investigación mediante encuestas. De hecho, hace ya unos años, García Ferrando recomendaba realizar más investigación sobre el trabajo de campo cuando señalaba -en 1979 - que los desarrollos producidos en los últimos años se habían destinado al análisis de los datos, dejando «en un lugar secundario la búsqueda de soluciones a los problemas de los métodos y técnicas de producción y obtención de datos» (García Ferrando, 2000: 130).

\section{Referencias bibliográficas}

BIEMER, P. P., y LYBERG, L. E. (2003): Introduction to Survey Quality, Nueva York, Wiley.

BRADBURN, N. M. (1992): «A Response to the Nonresponse Problem», Public Opinion Quarterly, vol. 56, pp. 391-397.

21 En nuestro caso, fue necesaria la ayuda de un inglés nativo para comprender algunas de las partes del vídeo. Aprovechamos esta situación para mostrar nuestro agradecimiento a George Buchan. 
CAMACHO, C.; PRADO, C.; ROMERO, B., y VALERA, S. (2000): “¿Cómo hacen encuestas los encuestadores», Metodología de Encuestas, vol. 2, n.ำ 1, pp. 117-133.

CAMPANELLI, P.; STURGIS, P., y PURDON, S. (1997): Can you hear me knocking: An investigation into the impact of interviewers on surveys response rates, Londres, SCPR.

CUXART, A., y RIBA, C. (2005): «Aspectos metodológicos de la Encuesta Social Europea», en M. Torcal, L. Morales y S. Pérez-Nievas, España: Sociedad y política en perspectiva comparada, Valencia, Tirant lo Blanch, pp. 21-40.

DÍAZ DE RADA, V. (2000): Problemas originados por la no respuesta en investigación social: Definición, control y tratamiento, Pamplona, Universidad Pública de Navarra.

- (2001a): Organización y gestión de los trabajos de campo con encuestas personales y telefónicas, Barcelona, Editorial Ariel.

- (2001b): «Estrategias para Incrementar la Tasa de Respuesta en las Investigaciones con encuestas», Revista Internacional de Sociología, n.ำ 29, pp. 133-162.

- (2004): «Exactitud de algunos prejuicios atribuidos a la encuesta postal: resultados de varias investigaciones», comunicación presentada en el III Congreso de Metodología de Encuestas, 15-17 de septiembre, Facultad de Sociología, Universidad de Granada.

- (2005): Manual del trabajo de campo en la encuesta, Ma-

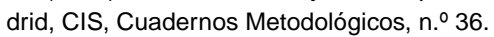

FERNÁNDEZ ESQUINAS, M. (2003): «Criterios de calidad en la investigación social: la producción de datos sociales», Empiria, n.ำ 6 , pp. 47-77.

GARCÍA FERRANDO, M. (2000): «La observación científica y la obtención de datos sociológicos", en M. García Ferrando, J. Ibáñez y F. Alvira (eds.), El Análisis de la Realidad Social, Madrid, Alianza, 3. ${ }^{a}$ ed., pp. 129-143 (edición original, 1986).

GROVES, R. M., y COUPER, M. (1998): Nonresponse in Household Interview Surveys, Nueva York, Wiley.

GROVES, R. M.; DILLMAN, D. A.; ELTINGE, J. L., y LITTLE, R. J. A. (2002): Survey Nonresponse, Nueva York, Wiley.

HOX J., y DeLEEUW, E. (2002): «The Influence of Interviewers'Attitude and Behavior on Household Survey Nonresponse: An International Comparison», en R. M. Groves et al. (eds.), Survey Nonresponse, Nueva York, Wiley, pp. 103-120.
INSTITUTE FOR SOCIAL RESEARCH, SURVEY RESEARCH CENTER (1976): Interviewer's Manual: Survey Research Center, Ann Arbor, Institute for Social Research, Universidad de Michigan (edición original, 1969).

KISH, L. (1949): «A procedure for objective respondent selection within the household", Journal of the American Statistical Association, vol. 44, pp. 92-115.

- (1965): Survey Sampling, Nueva York, Wiley; versión española en 1972, Muestreo de encuestas, Méjico, Trillas.

MANZANO, V.; ROJAS, A. J., y FERNÁNDEZ, J. S. (1996): Manual para encuestadores, Madrid, Ariel.

MORTON-WILLIAMS, J. (1991): Obtaining co-operation in surveys- the development of a social skills approach to interviewer training in introducting surveys, Join Centre for Survey Methods, Social and Community Plannig ResearchSCPR, Working Paper n. $\stackrel{0}{3}$.

NATIONAL CENTRE FOR SOCIAL RESEARCH (1999a): How to Improve Survey Response Rates. A guide for Interviewers on the Doorstep (vídeo), Londres, Sage.

- (1999b): How to Improve Survey Response Rates. A guide for Interviewers on the Doorstep (guía de utilización del vídeo), Londres, Sage.

- (1999c): Interviewers' Manual, Londres, National Centre for Social Research.

PARK y otros (2001): British Social Attitudes, Londres, Sage.

SÁNCHEZ CARRIÓN, J. J. (2000): La bondad de la encuesta: el caso de la no respuesta, Madrid, Alianza.

SMITH, T. W. (1995): “Trends in Non-Response Rates», International Journal of Public Opinion Research, vol. 7, pp. 157-171.

STATISTICS SWEDEN (1999): Journal of Official Statistics, Special issue on Survey Nonresponse, vol. 15, n.ำ 2 .

- (2001): Journal of Official Statistics, Special issue on Survey Nonresponse, vol. 17, n. $\stackrel{2}{2}$.

STOOP, I. A. (2005): The hunt for the last respondent. Nonresponse in sample surveys, The Hague, Social and Cultural Planning Office of the Netherlands.

WERT, J. I. (1996): Carta abierta a un incrédulo sobre las encuestas y su muy disputado crédito, Madrid, Península.

Vidal DÍAZ DE RADA 